\section{LENGUAJE, ONTOLOGÍA Y RELACIONES INTERTEÓRICAS: EN FAVOR DE UN GENUINO PLURALISMO ONTOLÓGICO}

\author{
Olimpia Lombardi \\ CONICET - Universidad de Buenos Aires, Argentina
}

Ana Rosa Pérez Ransanz

Instituto de Investigaciones Filosóficas, UNAM, México

\section{LANGUAGE, ONTOLOGY AND INTER-THEORETICAL RELATIONSHIPS: IN SUPPORT OF A GENUINE ONTOLOGICAL PLURALISM}

\begin{abstract}
The aim of this paper is to discuss the problem of intertheoretical relationships from the perspective of an ontological pluralism based on a Kantian rooted realism. For this purpose we begin by recalling the classical concept of reduction and its shortcomings, stressing the metaphysical assumptions involved in such conception. Next, we analyze another outspread position that conceives intertheoretical relations in terms of functional relationships, in order to underline certain misguided conclusions usually drawn from that kind of relationship. This task leads us to point out a problem usually ignored in these discussions, the problem about the way in which the system under study is defined. Finally, we introduce a general characterization of our Kantian rooted ontological pluralism, and try to show how this pluralism, in its synchronic version, allows us to face the problem of inter-theoretical relations in a philosophically fruitful way.
\end{abstract}

KEY WORDS: Inter-theoretical relationships; reduction; emergence; functional relationships; ontological pluralism; Kantian realism.

\section{El CONCEPTO TRADICIONAL DE REDUCCIÓN}

Bajo la influencia del giro lingüistico del siglo XX, en la filosofía clásica de la ciencia se abordó la cuestión de las relaciones entre teorías casi exclusivamente en términos del concepto de reducción. Sin embargo, mucho se ha discutido sobre el tema desde los trabajos pioneros de Ernst Nagel, y actualmente resulta difícil encontrar un autor que continúe anclado al concepto tradicional de reducción. En especial, la reflexión filosófica sobre las ciencias particulares nos ha venido mostrando que el modelo deductivista clásico de reducción carece prácticamente de aplicaciones en el desarrollo teórico efectivo de las disciplinas científicas.

\begin{abstract}
RESUMEN: En este artículo nos proponemos discutir el problema de las relaciones interteóricas desde la perspectiva de un pluralismo ontológico que adopta un realismo de raigambre kantiana. Para ello comenzamos por recordar el concepto tradicional de reducción y sus limitaciones, poniendo el énfasis en los supuestos metafísicos que conlleva tal concepción. A continuación, analizamos otra difundida postura que concibe las relaciones interteóricas en términos de relaciones funcionales, a fin de poner al descubierto ciertas conclusiones inadecuadas que suelen inferirse de tal interpretación. Esta tarea nos conduce a señalar un problema generalmente ignorado en las discusiones sobre el tema, el cual se refiere al modo como se define el sistema bajo estudio. Finalmente, presentamos una caracterización general de nuestro pluralismo ontológico de filiación kantiana, e intentamos poner de manifiesto cómo, en su versión sincrónica, tal pluralismo nos permite afrontar el problema de las relaciones interteóricas de un modo filosóficamente fecundo.
\end{abstract}

PALABRAS CLAVE: Relaciones interteóricas; reducción; emergencia; relaciones funcionales; pluralismo ontológico; realismo kantiano.

Según el enfoque clásico (Nagel, 1961), la reducción es una relación lógica entre teorías: la teoría reducida se deduce de la teoría reductora, con ayuda de ciertas definiciones que conectan los términos de ambas teorías $y$, en ocasiones, con algunas condiciones auxiliares singulares. Cuando lo único que se agrega a la teoría reductora es un conjunto de enunciados analíticos, o bien de condiciones contingentes, la teoría reducida no agrega contenido nomológico alguno a la teoría reductora. Según Nagel, un ejemplo paradigmático de esta situación es el de la reducción de la termodinámica a la mecánica estadistica, por lo cual el término "temperatura" de la termodinámica puede reducirse definicionalmente a términos de la mecánica 
estadistica: la temperatura se identifica con el valor medio de la energía cinética de las moléculas que componen un gas (Nagel, 1961, Cap. 11).

En la concepción nageliana, los principales vínculos interteóricos se establecen por definición: los términos de la teoría reducida que no aparecen en la teoría reductora se identifican definicionalmente con algún término 0 combinación lógica de términos de la teoría reductora. Y dado que la relación que se establece entre los miembros de una definición es la identidad lógica, ambos miembros refieren a un mismo item extralingüístico. Así, la identidad entre "temperatura" y "energía cinética media" implica que ambos términos designan el mismo ítem: la temperatura de un gas no es más que la energía cinética media de sus moléculas.

Este modo de concebir las relaciones interteóricas se encuentra en total sintonía con el realismo metafísico tradicional, el cual postula la existencia de una única ontología fundamental, por lo cual sólo podría caber una única descripción objetiva de tal ontología: las únicas entidades, propiedades y relaciones realmente existentes son aquéllas que pertenecen a la ontología de la teoría reductora. Los términos que introduce la teoría reducida carecen de referentes ontológicamente independientes: sólo son medios económicos para decir lo mismo que, en principio, podría decirse sin ellos. Por ejemplo, si un gas no es más que un conjunto de partículas en interacción, y su temperatura no es más que el valor medio de la energía cinética de tales partículas, entonces las palabras "gas" y "temperatura" no tienen otro referente que el que les brinda la teoría mecánica microscópica. Como afirma Lawrence Sklar para el caso de la reducción de la óptica física al electromagnetismo: "las ondas de luz no están correlacionadas con las ondas electromagnéticas, son ondas electromagnéticas" (Sklar, 1967, 120, énfasis nuestro).

Puesto que la visión deductivista de la reducción es una postura eliminativista, si existieran casos científicos en los que efectivamente pudieran establecerse tales nexos reductivos, tendriamos buenas razones para aplicar la navaja de Ockham y aceptar que la ontología está poblada exclusivamente por los ítems de los que nos habla la teoría reductora. Sin embargo, la aplicabilidad del modelo deductivo se ha visto severamente cuestionada durante las últimas décadas. Muchos autores han enfatizado que los ejemplos de reducción "à la Nagel" son triviales y no están bien fundamentados, e incluso se ha alegado que no existen casos científicamente relevantes que se ajusten al modelo deductivo (cfr. Feyerabend, 1962; Sklar, 1967; Scerri \& McIntyre, 1997; Primas, 1998). En efecto, a pesar de que los únicos nexos interteóricos que tradicionalmente se consideraron relevantes $-y$ dignos de estudio- fueron los de tipo reductivo, con el tiempo se ha ido encontrando que muchos de los nexos catalogados originalmente como reductivos involucran aproximaciones y otros procedimientos matemáticos mucho más complejos que la simple deducción lógica.

No obstante, aun entre quienes cuestionan la reducción nageliana, el debate se suele circunscribir al plano semántico cercenado de sus implicaciones ontológicas, por lo cual las discusiones básicamente giran en torno a las operaciones matemáticas que permiten establecer vínculos formales entre teorias. El hecho, en breve, es que la cuestión ontológica apenas se discute. A nuestro juicio, la principal razón se encuentra en el arraigado compromiso metafísico con una ontología última y fundamental, a la que correspondería la descripción verdadera y completa del mundo. De aqui que, se reconozca o no, siga perviviendo una suerte de reduccionismo ontológico que, entre otras cosas, conduce a catalogar a la mecánica cuántica como una teoria "fundamental", relegando a la termodinámica como una teoría meramente "fenomenológica"; y en la misma línea de pensamiento, la química resulta una disciplina "secundaria" frente a una ciencia como la física que se ocupa de los componentes últimos de la realidad. Las llamadas teorías "fundamentales" serían, entonces, aquéllas que supuestamente describen la realidad tal como es en sí misma, mientras que las teorías "fenomenológicas" o las disciplinas "secundarias" sólo describirían los hechos tal como se nos aparecen.

\section{Relaciones funcionales como nexos INTERTEÓRICOS}

Frente a los repetidos fracasos de la reducción interteórica clásica, algunos autores propusieron reemplazar la idea de definición por la idea de relación funcional y, con ello, el requisito de deducción lógica por el de deducción matemática. Desde esta perspectiva, ciertos términos de la teoría 
reducida se asimilan a funciones matemáticas de algunos términos de la teoría reductora. Esta idea es la que parece estar detrás de algunas versiones de la concepción estructuralista de reducción (por ejemplo, Stegmüller, 1973), cuando se establecen relaciones de muchos a uno entre las clases de modelos de las teorías reducida y reductora. Sin embargo, ya en su discusión con Sneed y Stegmüller, Kuhn (1976) ponía de relieve los problemas que plantea esta propuesta al señalar que la relación funcional, cuando se establece entre teorias inconmensurables, presupone una identificación entre conceptos básicos de dichas teorías, siendo que el problema consiste precisamente en justificar tal identificación. Aquí retomamos la línea de estos cuestionamientos de Kuhn, sobre la base de nuevos argumentos.

Para un físico, el ejemplo paradigmático de relación funcional interteórica es el nexo entre la energía cinética media por molécula en un sistema de moléculas, $\bar{E}_{1}$ y la temperatura $T$ del sistema concebido como un gas: $T=(2 /$ ek) $\bar{E}_{\mathrm{k}}=f\left(\bar{E}_{\mathrm{k}}\right)$, donde $k$ es la constante de Boltzmann. En general, una relación funcional interteórica puede expresarse como $Y=f\left(X_{1}, \ldots, X_{n}\right)$, donde $Y$ es un término de una teoría $\mathrm{T}_{2}, X_{1}, \ldots, X_{\mathrm{n}}$ son términos de una teoría $\mathrm{T}_{1}$, y $f$ expresa una función matemática. De esta manera, podría parecer que con este tipo de relación no nos alejamos demasiado de la concepción reductivista tradicional: también aquí tenemos un término que se "identifica" con una combinación, ahora funcional, de otros términos. Sin embargo, un examen más detenido de este tipo de expresiones mostrará que no es el caso.

En primer lugar, el símbolo "=" que aparece en las expresiones que comúnmente utiliza la física no expresa una identidad lógica. Esto puede comprobarse fácilmente en el caso de expresiones que representan leyes fundamentales de una teoría particular. Por ejemplo, cuando de acuerdo con la segunda ley de Newton se afirma " $F=m a$ ", no se pretende decir que " $F$ " y " $m a$ " designan un mismo ítem: " $F$ " denota la fuerza aplicada sobre un cuerpo, y " $m$ " y " $a$ " denotan la masa y la aceleración de dicho cuerpo respectivamente; los tres ítems son ontológicamente diferentes y los tres pueblan el mundo de la mecánica clásica. En nuestro ejemplo paradigmático de relación funcional interteórica, la única diferencia consiste en que el término " $\bar{E}_{\mathrm{K}}$ " pertenece a una teoría, la mecánica estadística, y el término " $T$ " pertenece a otra teoría, la termodinámica; no obstante, al igual que en el caso de la expresión de leyes, el símbolo "=" no nos dice que " $T$ " y " $(2 / 3 k) \bar{E}_{\mathrm{k}}$ " refieren a una misma entidad.

En segundo lugar, la función representada por " $f$ " es una función matemática, que especifica qué operaciones algebraicas deben efectuarse entre los valores numéricos que adoptan las propiedades correspondientes. Volviendo al ejemplo de la segunda ley de Newton, " $F=m a "$ nos dice que el valor numérico de la fuerza coincide con el número que se obtiene como producto entre el valor numérico de la masa y el valor numérico de la aceleración, expresados todos ellos en ciertas unidades de medida preestablecidas. Es claro que esto nada tiene que ver con identificar conceptos: el concepto "fuerza" no se identifica con el producto de los conceptos "masa" y "aceleración" por la sencilla razón de que no tiene sentido hablar de "multiplicación de conceptos". Y si esto es así en el caso de una relación funcional intrateórica, no hay motivo alguno para suponer que las expresiones de la física deban interpretarse de un modo diferente en el caso de relaciones funcionales interteóricas. Volviendo a nuestro ejemplo paradigmático, " $T=(2 / 3 k) \bar{E}_{K}=f\left(\bar{E}_{K}\right)$ " nos dice que el valor numérico de la temperatura de un gas contenido en un recipiente coincide con el número que se obtiene de multiplicar el valor numérico de la energía cinética media por molécula de las moléculas contenidas en el recipiente por 2/3, y luego dividirlo por el valor numérico de la constante de Boltzmann. La diferencia con el caso anterior es que aquí el concepto de "energía cinética media por molécula" pertenece a la mecánica estadística y, por tanto, su valor numérico debe obtenerse con los medios teóricos y empíricos de tal teoría, mientras que el concepto de "temperatura" pertenece a la termodinámica y su valor numérico se obtiene por medición con las técnicas e instrumentos que esta teoría presupone. Pero, a pesar de la coincidencia numérica, los conceptos son genuinamente diferentes $y$, en consecuencia, refieren a ítems ontológicos irreductibles. Como afirma Fritz Rohrlich (2001, 193): "No puede haber un mejor ejemplo de inconmensurabilidad ontológica que energía cinética y temperatura".

Los argumentos anteriores muestran que si bien las relaciones funcionales interteóricas, al incluir en su forma el símbolo "=", pudieran sugerir algún tipo de identificación conceptual, sin embargo no deben interpretarse en ese sentido. En la expresión " $Y=f\left(X_{1}, \ldots, X_{n}\right)$ " tenemos que 
" $Y$ " denota un concepto de la teoría $T_{2}, " X_{1}$ ", ..., " $X_{n}$ " denotan conceptos de la teoria $T_{1}$, y la igualdad matemática sólo indica una coincidencia entre valores numéricos. El concepto denotado por " $Y$ " puede no existir en la teoría $\mathrm{T}_{1}, \mathrm{y}$ los conceptos denotados por " $X_{1}$ ", ..., " $X_{n}$ " pueden no existir en la teoría $T_{2}$. Cada teoría refiere a su propia ontología y la coincidencia entre valores numéricos no cancela este hecho. No son, entonces, las expresiones de la propia física, sino los presupuestos de una vieja metafísica lo que conduce a establecer nexos asimétricos entre dichas ontologías, esto es, a confinar una de ellas al ámbito de lo subjetivo o aparente, o a considerarla como secundaria o dependiente respecto de la otra. Por otra parte, cabe destacar que la coincidencia entre valores numéricos no resulta de una definición y tampoco expresa una verdad lógicamente necesaria, simplemente expresa una regularidad legal que no establece prioridad alguna entre sus términos. En efecto, en la medida en que el valor numérico de $Y$ se obtiene mediante la teoría $T_{2}$, y los valores numéricos de $X_{1}, \ldots, X_{n}$ se obtienen mediante la teoría $\mathrm{T}_{1}$, la igualdad numérica expresada por " $Y=f\left(X_{1}, \ldots, X_{n}\right)$ " posee un contenido nomológico $y$, por lo tanto, su valor de verdad debe ser establecido por la ciencia empírica y no por la lógica.

En definitiva, las relaciones funcionales interteóricas establecen nexos legales entre teorias, nexos que, en principio, no cancelan la independencia ontológica entre sus respectivos dominios de referencia. Quien, a pesar de ello, pretenda insistir en la prioridad ontológica de un dominio respecto del otro, deberá asumir tal posición como un supuesto metafísico explícito, sin presentarla como una consecuencia de la relación formal entre las teorías correspondientes.

\section{LA DEFINICIÓN DEL SISTEMA: UN PROBLEMA IGNORADO}

Al estudiar una situación particular, el primer paso hacia una representación o descripción científica consiste en delimitar el sistema bajo estudio. Este aspecto, que suele ser ignorado al abordar la cuestión de las relaciones interteóricas, resulta esencial para la discusión del problema que aquí nos ocupa, ya que sólo tiene sentido preguntarse por las relaciones de reducción o subsunción entre teorías que brindan descripciones diferentes de un mismo sistema.
De aquí que nadie se pregunte por este tipo de relaciones entre una teoría biológica y una teoría cristalográfica, o entre la mecánica de fluidos y la teoría de semiconductores. En cambio, la pregunta tiene pleno sentido entre teorias como la termodinámica y la mecánica clásica, en la medida en que se supone que ambas teorías describen de modos diferentes un mismo sistema físico.

No es posible en física, y quizás tampoco en otras disciplinas científicas, definir con total generalidad la noción de "sistema", puesto que tal noción funciona prácticamente como un concepto primitivo. No obstante, podemos preguntarnos por los criterios que utilizan los científicos para delimitar el sistema bajo estudio. Si prestamos atención a la práctica efectiva, comprobaremos que aunque un sistema suele identificarse por su ubicación espacial y temporal, o por su aislamiento -suficiente pero nunca perfecto- respecto del entorno, sin embargo ninguno de estos criterios resulta totalmente general. En muchos casos, en una misma región del espacio y en un mismo intervalo de tiempo, ciertas variables o grados de libertad se consideran como constitutivos del sistema, y el resto como parte del entorno. Por ejemplo, en la teoría actual de la decoherencia cuántica, el sistema se identifica con los grados de libertad que corresponden a ciertas variables "colectivas" (Omnès, 1999) o "relevantes" (Castagnino, Laura \& Lombardi, 2007), que luego de un intervalo ínfimo -el tiempo de decoherencia- adquieren un comportamiento aproximadamente clásico. En cosmología, una estrategia usual consiste en dividir el universo en ciertos grados de libertad que representan el sistema de interés, mientras que los restantes, al suponerse como no accesibles, funcionan como un entorno "interno". En teoría cuántica de campos, dado un campo escalar $f$, éste suele descomponerse como $f=f_{\mathrm{S}}+f_{\mathrm{E}}$ donde $f_{\mathrm{S}}$ denota el campo del sistema y $f_{\mathrm{E}}$ denota el campo del entorno. Pero lo importante en estos casos es que no existe un único criterio para efectuar tal descomposición: en ocasiones el campo se descompone sobre la base de escalas de longitud, masa o momento cinético; otras veces el sistema se define como los campos con una frecuencia menor que un cierto valor, y los campos de mayor frecuencia se consideran como el entorno (cfr. Calzetta et al., 2001).

Estos casos, como muchos otros de la práctica científica, ponen de manifiesto que desde el primer paso de la tarea descriptiva opera necesariamente algún sistema de con- 
ceptos que permite fijar ciertos items particulares (por ejemplo, espacio y tiempo) o ciertas propiedades básicas (como posición espacial, ubicación temporal, temperatura, velocidad, frecuencia, etc.), mediante los cuales el sistema se identifica y se "desprende" del entorno. En otras palabras, no existen sistemas "autoidentificantes", ya que nada hay en un campo de estudio que imponga cuál es el sistema y cuál es el entorno, con independencia de la intervención de una teoría con su propio sistema de conceptos. Este hecho está implicitamente reconocido en la práctica misma de la física: cualquier físico admite que no hay sistemas-en-sí, sino que los sistemas que pueblan su ontología resultan de un "recorte", de una primera distinción conceptual que depende de la teoría utilizada.

Este hecho, tan básico y familiar, prácticamente se ha pasado por alto en las discusiones tradicionales acerca de las relaciones interteóricas. En el modelo deductivista clásico, se da por supuesto que la identidad de los sistemas estudiados es lógicamente previa a las teorías que los intentan explicar. Ya sea que se los conciba como algo "dado" en la experiencia, o como independientes de toda determinación conceptual, se da por hecho que los sistemas están alli, a la espera de ser descubiertos y explicados por las diferentes teorias. Este supuesto metafísico, $\tan$ claramente reñido con la manera como se usan las teorías en una ciencia tan "fundamental" como la física, bien puede ser abandonado sobre la base de una perspectiva filosófica distinta: una perspectiva que dé cabida a un genuino pluralismo ontológico, sin que ello implique renunciar al realismo, pero donde se reconozca de entrada el carácter conceptualmente constituido de todo objeto de conocimiento (de aquí su filiación kantiana).

\section{Plutalismo ontológico de raigambre kantiana}

Antes de Kant, la polémica giraba en torno de si hay realmente objetos "ahí afuera", objetos físicos o materiales, o si sólo existe lo mental. Kant comienza por rechazar los supuestos de esta discusión y plantea el problema del realismo en otros términos. No se trata de establecer qué es lo que realmente existe, el problema estriba en aceptar que todo objeto de conocimiento, del tipo que sea, está constituido en el marco de nuestro esquema categorial y es, por tanto, "objeto para nosotros". Kant nunca pone en duda la existencia de una realidad independiente del sujeto; para él, esto es un postulado de la razón. Incluso argumenta que podemos atribuir a dicha realidad la capacidad de constreñir y cuestionar nuestros juicios empíricos. Sin embargo, le interesa destacar que, a pesar de ello, no nos podemos formar ninguna idea o concepto de "lo nouménico" o "cosa-en-si".

De este modo, Kant se aleja tanto del idealismo como del realismo trascendental (realismo metafísico). Contra el idealismo, señala repetidamente que el propio término "fenómeno" conlleva una referencia a algo distinto de nuestras representaciones, un algo que interviene necesariamente en la constitución del objeto de conocimiento:

el carácter puramente fenoménico de los objetos de la experiencia no excluye sino más bien implica una realidad trascendente que les sirve de base y que, aunque incognoscible, no es por ello menos efectiva. [...] los objetos fenoménicos no son meros fantasmas insustanciales, [...] la percepción en que se manifiesta su presencia revela una existencia efectiva (Torretti, 2005a, 676-677, énfasis nuestro).

De aquí el carácter genuinamente realista de su posición. En cuanto al realismo metafísico, Kant es muy claro cuando insiste en el carácter incognoscible de lo nouménico. Como afirma Torretti, no sólo no hay una correspondencia biunivoca entre cosas-en-sí y objetos de la experiencia, sino que tampoco puede suponerse que cada una de las determinaciones de los objetos empíricos encuentra su razón de ser en la cosa-en-sí. Quien asumiera este supuesto tendría que admitir que "Kant ha derogado de una plumada toda su enseñanza acerca de la limitación de nuestro conocimiento" (Torretti, 2005a, 664-665).

El Putnam de los años 80 reconoce que su "realismo internalista" está muy cerca de la filosofía de Kant, y rechaza el realismo metafísico (externalista) por las mismas razones que Kant, en su momento, rechazara el realismo trascendental. Su primer blanco de ataque es la noción de objeto como algo que tiene una identidad propia pero a la vez existe con total independencia de nuestro conocimiento (mente, lenguaje, representaciones o esquemas conceptuales). Esto es, rechaza la idea de objeto autoidentificante. Y la razón que aduce, en breve, es que: "Los 
'objetos' no existen independientemente de los esquemas conceptuales. Desmenuzamos el mundo en objetos cuando introducimos uno u otro esquema descriptivo" (Putnam, 1981, 52). Es siempre a través de un cierto sistema de conceptos que interactuamos con el mundo y lo categorizamos, y es de tal sintesis que surgen los objetos en cuanto tales, es decir, con una identidad.

Ahora bien, aun cuando Kant "compara atinadamente al científico con un juez instructor que dirige sus preguntas a la naturaleza y fija los términos en que tiene que venir concebida la respuesta, no se pone [nunca] en el caso de que, frustrado porque las respuestas se contradicen, las indagaciones se complican y atascan, y en general no parecen estar llegando a nada, el juez instructor repiense sus preguntas, modifique las categorias en que deben encuadrarse las respuestas e incluso cambie las metas de la investigación o los criterios para evaluar sus resultados" (Torretti, 2005b). Por ello, a la noción kantiana de objeto, como objeto conceptualmente constituido, el Putnam historicista le agrega la tesis de la relatividad conceptual, según la cual ningún concepto -ni siquiera las categorías más básicas- tiene una interpretación única 0 absoluta. No hay un concepto privilegiado de objeto, ni de existencia, que sea el metafísicamente correcto. De aquí que coexistan esquemas conceptuales alternativos, no convergentes ni reducibles a un esquema único. La idea de que la realidad nos impone una correspondencia única entre nuestros conceptos y las cosas, como si hubiera una especie de pegamento metafísico entre lenguaje y mundo, es una mera ilusión. El fenómeno de la relatividad conceptual "depende del hecho de que los mismos primitivos lógicos, y en particular las nociones de objeto y existencia, tienen una multitud de usos diferentes y no un 'significado' absoluto" (Putnam, 1987, 19).

Asi entendida, la tesis de la relatividad conceptual trae consigo un pluralismo ontológico, pues abre la posibilidad de tener concepciones del mundo con ontologías distintas -incluso incompatibles- que resulten igualmente adecuadas en ciertos contextos, en función de los intereses y objetivos en juego. De aquí que la pregunta sobre qué es lo que hay en el mundo requiera de la especificación del esquema conceptual desde el cual se plantea e intenta responder. Sólo cuando hemos adoptado un sistema de conceptos podemos afirmar que, en efecto, algunos hechos y objetos están ahí para ser descubiertos. En otras palabras, sólo desde el plano del conocimiento, el plano de nuestras representaciones, tiene sentido preguntarnos por aquello que existe. De aquí que la crítica de fondo al realismo metafísico se dirija a su compromiso con categorías ontológicas absolutas y, por tanto, con una estructura de la realidad que sólo podría coincidir con la del punto de vista del Ojo de Dios.

En este punto no podemos dejar de mencionar la meticulosa crítica que hace C.U. Moulines al realismo metafísico (que él denomina "referencial"), en la que toma como punto de partida la inconmensurabilidad entre teorías. Moulines muestra cómo esta relación interteórica se sigue de manera natural del principio de inescrutabilidad de la referencia postulado por W.V. Quine, junto con otras tesis clave de este autor como la indeterminación de la traducción y el holismo semántico (cfr. Moulines, 1991, II.2.2). Por esta vía, en una línea de argumentación similar a la de Putnam pero reforzada con tesis adicionales, Moulines sostiene que la consecuencia que obtiene Quine en La relatividad ontológica (1969) es que "la noción de referencia, tomada como algo absoluto, carece de sentido", ya que: "No hay un punto de vista supralingüístico absoluto desde el cual poder fijar la referencia de cada término con independencia de sus relaciones con otros términos" (Moulines, 1991, 148). Por tanto, la referencia de un término sólo puede fijarse en el interior de un marco lingüistico o conceptual.

Por otra parte, también cabe señalar que el deslinde entre pluralismo y relativismo, que tanto preocupa a los defensores de posiciones pluralistas, es un deslinde bastante sencillo de establecer. Como señala Moulines, si bien la actitud anti-absolutista que comparten el pluralismo y el relativismo los acerca peligrosamente, sin embargo, en la medida en que se tengan claros los objetivos y metas que se persiguen en cada contexto, podemos disponer de criterios efectivos de evaluación de las alternativas existentes -se trate de sistemas de creencias o de métodos-, en función de su mayor o menor adecuación a dichos objetivos. El pluralismo, a diferencia de los relativismos perniciosos, admite criterios objetivos de evaluación (pues no todas las alternativas son equiparables). Pero a diferencia de los absolutismos fundamentistas, se opone a la idea de un único sistema válido de creencias (o de métodos) en el plano del conocimiento humano (cfr. Moulines, 1991, 30-31). 


\section{El SISTEMA COMO OBJETO DE CONOCIMIENTO}

En sus trabajos de los años 80, también en la línea trazada por Quine (1969), Putnam contempla la posibilidad de brindar un sentido sincrónico al pluralismo ontológico, en particular cuando se refiere a la coexistencia de diferentes ontologías o "mundos", como serían los mundos del sentido común y de las diferentes teorías científicas vigentes en un momento dado. No obstante, la diversidad de mundos ha jugado su papel más relevante en el análisis diacrónico del cambio de teorias, justo a partir de las tesis de Kuhn (1962). Desde entonces, se ha esgrimido como el principal argumento contra el realismo metafísico de corte cientificista, según el cual las ontologías de teorías sucesivas convergen hacia la descripción verdadera y completa de los componentes últimos de la realidad, una especie de superteoría que serviría de marco referencial universal. Sin embargo, cuando el objetivo consiste en analizar el problema de las relaciones interteóricas, sin restringirse a una perspectiva meramente semántica sino atendiendo a los vínculos entre las ontologías de teorias científicas vigentes (o coexistentes), el sentido sincrónico del pluralismo ontológico cobra toda su relevancia.

Como hemos visto, en la práctica científica no existen sistemas "autoidentificantes"; nada hay en la naturaleza que nos imponga la identificación de un sistema con independencia de la teoría que se utiliza en su descripción. Este hecho no cuestionado, pero casi nunca explicitado, adquiere una formulación precisa en el realismo pluralista que defendemos: es mediante el esquema conceptual de la teoría utilizada como se efectúa una primera delimitación, un primer recorte del sistema que se individualiza, se identifica y se "desprende" del entorno. Por ejemplo, si el esquema conceptual contiene la categoría de objeto individual y las propiedades de ubicación espacial y temporal como propiedades definitorias de dichos objetos, el sistema podrá identificarse por su ubicación espacial y temporal; si el esquema conceptual contiene campos como entidades básicas y la propiedad de frecuencia como propiedad definitoria de tales entidades, el sistema podrá definirse como un campo con una frecuencia menor que un cierto valor. Este operar teórico de la ciencia pone de manifiesto que los científicos, aun sin reconocerse como realistas de corte kantiano, de hecho actúan como tales cuando efectúan un primer recorte de la realidad, con el fin de distinguir entre el sistema bajo estudio y su entorno.
Una situación muy común en física es la que se da cuando dos teorias brindan descripciones diferentes de "un mismo sistema". En el caso en que presupongan el mismo esquema conceptual, ambas teorías recortan el mismo sistema sobre la base de las mismas categorias y conceptos, y la diferencia en las descripciones obedecerá simplemente a que hacen afirmaciones que son incompatibles; la tarea del científico será, entonces, contrastarlas empíricamente a fin de descartar una de ellas, o ambas.

Un caso más interesante se presenta cuando dos teorías establecidas, igualmente aceptadas por su éxito empírico, brindan no obstante descripciones diferentes del mismo sistema. En tal caso, dichas teorias comparten algunos elementos conceptuales, al menos aquéllos que les permiten establecer la misma primera identificación del sistema bajo estudio (a este respecto resulta muy útil el criterio de teoricidad propuesto por los estructuralistas para distinguir los elementos conceptuales propios de cada teoría; cfr. Balzer \& Moulines, 1980). Por ejemplo, los esquemas conceptuales de la termodinámica macroscópica y de la mecánica estadística clásica comparten los mismos conceptos de espacio y de tiempo, y sobre esta base puede identificarse el mismo sistema como aquello que ocupa una cierta posición espacial durante un cierto intervalo temporal. No obstante, dado que los esquemas conceptuales son diferentes, constituyen diferentes ítems ontológicos sobre el sistema previamente identificado: las entidades individuales, propiedades, relaciones, etc., de las que habla una teoría pueden no existir para la otra, y viceversa. Mientras que para la termodinámica el sistema es concebido como un gas, esto es, una entidad individual con propiedades como presión y temperatura, en cambio, el mismo sistema es descrito por la mecánica estadística como un conjunto de partículas -moléculas- en interacción, cada una de ellas con su propia masa y velocidad. Por tanto, en la ontología termodinámica no existen las partículas ni sus propiedades, como no existen gas ni temperatura en la ontología mecánico-estadística. Esta situación se repite en distintos ámbitos de la física, e incluso en la relación entre diferentes disciplinas científicas, como cuando se considera la coexistencia de una macro descripción química y una micro descripción mecánico-cuántica de un mismo sistema.

De este modo, hemos regresado al problema de las relaciones interteóricas, pero ahora reformulado en su dimen- 
sión ontológica: cómo concebir las ontologías referidas por descripciones distintas y cuál es la relación entre ellas. La dificultad se manifiesta con mayor claridad cuando una de las descripciones adjudica al sistema una cierta propiedad $\mathrm{P}$ y la otra le adjudica su negación $\neg \mathrm{P}$ : ¿cómo admitir predicaciones contradictorias sobre un mismo sistema? Además, el hecho de que esta situación se repita en diferentes ámbitos de la física, e incluso en la relación entre física y química, hace aún más aguda la dificultad. Por ejemplo, los sistemas dinámicos altamente inestables son deterministas bajo una descripción microscópica pero indeterministas en su descripción macroscópica. Lo mismo sucede en el debatido problema de la irreversibilidad: las evoluciones son reversibles, según la descripción microscópica, e irreversibles, según la descripción macroscópica. La situación reaparece en el límite clásico de la mecánica cuántica, donde el problema consiste en compatibilizar la existencia de objetos separables según las teorías clásicas con la ontología no separable de la mecánica cuántica.

En todos estos casos, el realista metafísico estará comprometido a decidir cuál de las propiedades es la "real": ¿es el sistema determinista o indeterminista?, ¿reversible o irreversible?, ¿separable o no separable? En general, el realista metafísico opta por asumir la perspectiva reduccionista clásica: incorporando un compromiso ontológico a la concepción semántica nageliana, supone que la teoría microscópica es la teoría "fundamental", la cual, por tanto, nos proporciona la descripción de la "verdadera" ontología. Si esto es así, determinismo, reversibilidad y no separabilidad serían las propiedades que "realmente" posee el sistema, y por tanto calificarían como propiedades intrínsecas de la realidad independiente. Pero esto implica nada menos que confinar al reino de la mera subjetividad todos los procesos indeterministas e irreversibles con los que nos enfrentamos cada día y, junto con ello, admitir el carácter meramente aparente o ilusorio de nuestra realidad clásica separable.

Estas conclusiones, que suelen entrar en conflicto con nuestras intuiciones más básicas, tanto de sentido común como cientificas, pueden evitarse desde un pluralismo ontológico como el que aquí presentamos. En efecto, cuando se admite que el esquema conceptual supuesto por cada teoría constituye, al menos en parte, la ontología a la cual cada teoría refiere, no hay inconveniente en sostener que tanto la propiedad $\mathrm{P}$ como la propiedad $\neg P$ son reales y objetivas en sus respectivos mundos. Ya no necesitamos confinar al ámbito de la mera subjetividad aquello de lo que nos habla un considerable número de teorias científicas actualmente vigentes. Puesto que cada esquema contribuye a la constitución de su propia ontología, no hay contradicción al adjudicar propiedades incompatibles a ontologías diferentes.

En cuanto a los casos de inconmensurabilidad extrema como los esgrimidos por Feyerabend (1962), donde las teorias que pretenden hablar de "lo mismo" no comparten, sin embargo, ninguna categoría o concepto de clase $y_{\text {, en }}$ consecuencia, la intersección de sus ámbitos de referencia resulta vacia, remitimos al lector al análisis que desarrolla Moulines (1991, 142-147). Para los propósitos del presente trabajo nos bastan los casos menos radicales, donde las teorias comparten un mínimo de su aparato conceptual, ya que además son los casos sobre los cuales giran las controversias que cotidianamente se dan entre los científicos -sin que medie revolución alguna-, controversias a cuya resolución o disolución intentamos contribuir con el realismo pluralista que aquí proponemos (una exposición más completa se encuentra en Lombardi \& Pérez Ransanz, 2007).

\section{HaCIA UNA IMAgen DIVERSIFICADA DE LO REAL}

Hace tiempo que un buen número de autores ha intentado evitar las conclusiones subjetivistas que se derivan del reduccionismo clásico, mediante el recurso a la noción de emergencia: cuando dos teorias brindan descripciones incompatibles de un mismo sistema, se considera que los items referidos por una de ellas "emergen" de los ítems referidos por la otra. Si bien el concepto de emergencia ha dado lugar a múltiples caracterizaciones, a menudo divergentes (EI Hani, 2004), en todas ellas puede reconocerse al menos una nota común: la relación de emergencia se concibe como una relación asimétrica. En efecto, si un item $A$ emerge a partir de un item (o conjunto de items) $B$, entonces $B$ no emerge de $A$. En el plano ontológico esto significa que si $B$ no existiera, tampoco existiria $A$, lo cual implica la prioridad ontológica del ítem $B$ y el carácter ontológicamente derivado del ítem A. Por lo tanto, aun sin negar la existencia objetiva del dominio emergente, el emergentismo sigue anclado a la idea de una jerarquía de 
teorias y ontologías, de las más a las menos fundamentales, por lo que la mecánica cuántica sigue prevaleciendo como una teoría "fundamental" y la termodinámica como una teoria meramente "fenomenológica". Y en el campo de las relaciones interdisciplinarias, la química permanece como una disciplina "secundaria" respecto de la física (una defensa de la autonomía de la química frente a la física se encuentra en Lombardi \& Labarca, 2005, 2006).

La pregunta que aquí se impone, entonces, remite a las razones para aceptar tal jerarquización: ¿cuál es la justificación para suponer una relación asimétrica entre dos dominios ontológicos? En nuestro análisis de las relaciones funcionales señalamos que, en principio, estas relaciones sólo indican una identidad entre valores numéricos y no una dependencia conceptual ni, por tanto, ontológica. Tal identidad expresa un vínculo legal totalmente simétrico que no establece prioridad alguna entre los correlata en cuestión. Por lo tanto, nada hay en el formalismo teórico que imponga nexos asimétricos entre las ontologías interconectadas por una relación funcional.

Por otra parte, a diferencia de lo que algunos autores suponen, las relaciones funcionales no son la única for- ma en que se manifiestan las relaciones interteóricas. Los vínculos entre teorías a menudo exigen técnicas matemáticas bastante más complejas, como, por ejemplo, el paso al límite y la introducción de grano grueso, donde también se observa que los nexos formales entre teorías no introducen, per se, asimetría alguna (cfr. Lombardi \& Pérez Ransanz, 2007). Por lo tanto, insistimos, si se pretende defender el carácter fundamental de ciertos dominios respecto de los restantes, tal posición deberá asumirse como un supuesto metafísico explícito, sin pretender que la prioridad ontológica se infiere o viene impuesta por las propias teorias o por el formalismo en que se expresa la relación entre ellas.

Por contraste, el realismo pluralista que proponemos permite abandonar la tradicional jerarquía que organiza las teorías y disciplinas científicas por su "distancia" respecto de ciertas teorías supuestamente "fundamentales". Desde esta perspectiva, la ciencia nos presenta una realidad diversificada que se despliega en una multiplicidad de ontologías, todas ellas igualmente objetivas $y$, en su mayoria, vinculadas mediante nexos que no necesariamente suponen identificaciones ni prioridades en el plano de lo existente.
Recibido: 21 de junio de 2009

Aceptado: 10 de diciembre de 2009

\section{BIBLIOGRAFÍA}

Calzetta, Esteban A., Bei Lok Hu y Francisco D. Mazzitelli (2001): "Coarse-Grained Effective Action and Renormalization Group Theory in Semiclassical Gravity and Cosmology", Physics Reports 352: 459-520.

Castagnino, Mario, Roberto Laura y Olimpia Lombardi (2007): "A General Conceptual Framework for Decoherence in Closed and Open Systems", Philosophy of Science, a aparecer en número a designar.

El Hani, Charbel N. (2004): "Os Debates sobre a Emergéncia de Propriedades: Onde Estamos Atualmente?", en Rabossi, Eduardo (ed.), La Mente y sus Problemas. Temas Actuales de Filo- sofía de la Psicología, Buenos Aires: Catálogos.

Feyerabend, Paul K. (1962): "Explanation, Reduction, and Empiricism", en Herbert Feigl and George Maxwell (eds.), Minnesota Studies in the Philosophy of Science Vol. 3, Dordrecht: Reidel, pp. 28-97.

Kant, Immanuel (1781): Critique of Pure Reason, traducción de N. Kemp Smith, Nueva York: St. Martin's Press, 1965.

Kuhn, Thomas S. (1976): "Theory-Change as Structure-Change: Remarks on the Sneed Formalism", Erkenntnis 10: 179-199.

Lombardi, Olimpia y Martín Labarca (2005): "The Ontological Autonomy of the Chemical World", Foundations of Chemistry 7: 125-148. 
Lombardi, Olimpia y Martín Labarca (2006): "The Ontological Autonomy of the Chemical World: A Response to Needham", Foundations of Chemistry 8: 81-92.

Lombardi, Olimpia y Ana Rosa Pérez Ransanz (2007): Los múltiples mundos de la ciencia (volumen en preparación).

Moulines, C. Ulises (1984): "Ontological Reduction in the Natural Sciences", en Balzer, Wolfgang, David A. Pearce y Heinz-Jürgen Schmidt (eds.), Reduction in Science, Dordrecht: Reidel, pp. 51-70.

Moulines, C. Ulises (1991): Pluralidad y recursión, Madrid: Alianza Editorial.

Nagel, Ernst (1961): The Structure of Science, New York: Harcourt, Brace \& World.

Omnès, Roland (1999): Understanding Quantum Mechanics, Princeton: Princeton University Press.

Pérez Ransanz, Ana Rosa (1999): Kuhn y el cambio científico, México: Fondo de Cultura Económica.
Pérez Ransanz, Ana Rosa y J. Francisco Álvarez (2004): "De Kant a Kuhn, acotando por Putnam", Éndoxa 18: 495-517.

Primas, Hans (1998): "Emergence in Exact Natural Sciences", en Farre, George L. y Tarkko Oksala (eds.), Acta Polytechnica Scandinavica 91: 83-98.

Putnam, Hilary (1981): Reason, Truth and History, Cambridge: Cambridge University Press. Los números de página corresponden a la versión castellana Razón, Verdad e Historia, Madrid: Tecnos, 1988.

Putnam, Hilary (1987): The Many Faces of Realism, La Salle, Illinois: Open Court. Los números de página corresponden a la versión española Las mil caras del realismo, Barcelona: Paidós, 1994.

Quine, Willard van Orman (1960): Word and Object, Cambridge, Mass.: The MIT Press.

Quine, Willard van Orman (1969): Ontological Relativity and Other Essays, New York: Columbia University Press.
Rohrlich, Fritz (2001): "Cognitive Scientific Realism", Philosophy of Science 68: 185-202.

Scerri, Eric R. y Lee Mclntyre (1997): "The Case for the Philosophy of Chemistry", Synthese 111: 213-232.

Sklar, Lawrence (1967): "Types of Intertheoretic Reduction", The British Journal for the Philosophy of Science 18: 109-12.

Stegmüller, Wolfgang (1973): Theorienstrukturen und Theoriendynamik, Berlin/Heidelberg: Springer (trad. castellana: Estructura y dinámica de teorías, Barcelona: Ariel, 1983).

Torretti, Roberto (2005a): Manuel Kant, Santiago de Chile: Ediciones Universidad Diego Portales.

Torretti, Roberto (2005b): "Conocimiento Discursivo", Lección Inaugural dictada en la Universidad Autónoma de Barcelona el 28 de abril de 2005, en ocasión de la investidura del autor como Doctor Honoris Causa de dicha Universidad. 